\title{
Planos de mobilidade corporativa: análise e proposta de método para sua elaboração
}

\author{
Guillermo Sant'Anna Petzhold ${ }^{1}$ e Luis Antonio Lindau²
}

\begin{abstract}
Resumo: Organizações desempenham um papel fundamental em questões atreladas ao transporte. Por volta de $50 \%$ dos deslocamentos diários nas cidades brasileiras ocorrem por motivo de trabalho. O conceito de mobilidade corporativa ainda é incipiente no país, embora difundido internacionalmente. A elaboração de planos de mobilidade corporativa estimula hábitos mais sustentáveis e eficientes de deslocamentos ao trabalho. Através da análise de 16 métodos de países onde o conceito já está estabelecido, propõe-se um roteiro adaptado à realidade local que conduza organizações no desenvolvimento desses planos. O método se diferencia em seis principais pontos frente aos analisados: (i) identificação da necessidade, (ii) mapeamento dos atores envolvidos, (iii) definição do escopo e reavaliação das metas iniciais, (iv) protagonismo da comunicação, (v) necessidade de cooperação técnica e (vi) coleta de dados sobre custos. Através do método proposto, espera-se influenciar organizações a desenvolverem planos de mobilidade corporativa que venham a beneficiar funcionários, as próprias organizações e as cidades.

Palavras-chave: gestão da demanda de viagens, deslocamento casa-trabalho, empresas.
\end{abstract}

\begin{abstract}
Institutions play a key role in issues related to transportation. In Brazilian cities, approximately $50 \%$ of daily trips are done for the purpose of work. The corporate mobility concept is still incipient in Brazil, although widely spread internationally. The development of corporate mobility plans stimulates more sustainable and efficient forms to travel to work. Through the analysis of 16 methods from countries where the concept is already established, we propose a roadmap adapted to the Brazilian reality, which helps organizations in the development of these plans. The method differs in six main points from those analyzed: (i) identification of the need, (ii) map of stakeholders, (iii) definition of scope and revision of initial targets, (iv) leading role of communication, (v) need for technical cooperation and (vi) data collection on costs. Through the proposed method, we expect to influence and help organizations in developing corporate mobility plans that will benefit employees, themselves and cities.
\end{abstract}

Keywords: travel demand management, commute to work, employers.

\section{INTRODUÇÃO}

Por muitos anos, cidades enfrentaram (e algumas ainda enfrentam) os problemas de mobilidade urbana com a construção de novas infraestruturas para aumentar a capacidade viária e assim acomodar o crescente número de automóveis. Esta estratégia tentou resolver problemas de mobilidade através da maior oferta de espaço viário. Contudo, a prática acabou por induzir o aumento de tráfego, do congestionamento e uma maior dependência do automóvel (Broaddus et al., 2009).

No âmbito corporativo, as questões de transporte resultam em uma série de problemas (Figura 1) como, por exemplo, maiores custos de transporte ou contínua necessidade de aumentar o número de vagas de estacionamento. $\mathrm{O}$ que muitas vezes não se percebe é que o setor corporativo possui um papel fundamental nas questões e na busca por soluções dos problemas relacionados ao transporte (Petzhold, 2016; Van Malderen et al., 2012).

Aproximadamente $50 \%$ de todos os deslocamentos realizados diariamente, nas grandes cidades brasileiras, têm como motivo o trabalho (Bahia, 2012; Distrito Federal,

\footnotetext{
1 Guillermo Sant'Anna Petzhold, Laboratório de Sistemas de Transportes, UFRGS. (guillermo.petzhold@wri.org)

2 Luis Antonio Lindau, Laboratório de Sistemas de Transportes, UFRGS.

(tlindau@wri.org)
}

Manuscrito recebido em 25/03/2016 e aprovado para publicação em 28/07/2016

Este artigo é parte de TRANSPORTES v. 25, n. 1, 2017. ISSN: 2237-1346 (online). DOI:10.14295/transportes.v25i5.1102
2010; Minas Gerais, 2013; Porto Alegre, 2004; Rio de Janeiro, 2015; São Paulo, 2012, 2013). Estes trajetos são responsáveis por consumir entre $55 \mathrm{~min}$ e $85 \mathrm{~min}$ diários (Pereira e Schawanen, 2013) ou de 10 a 15 dias por ano de cada funcionário (Petzhold e Lindau, 2015), além de implicar consideráveis despesas às pessoas.

Uma das principais causas para essa elevada perda de tempo é o fato de a maioria das organizações contar com uma jornada de trabalho com horários coincidentes de início e término, o que causa uma concentração de viagens em um curto período de tempo. Outro agravante que pode ser ressaltado é o fato da maior parte dos automóveis estar ocupada por uma única pessoa. Isso não só gera congestionamentos nos acessos e entorno dos locais de trabalho, como requer uma maior oferta de vagas de estacionamento, que tem um alto custo de construção e manutenção tanto para as organizações como para o setor público. Por fim, também devem ser considerados os impactos ambientais, pois $47 \%$ das emissões de $\mathrm{CO}_{2 \text { eq }}$ do setor de energia no Brasil são provenientes do transporte (Observatório do Clima, 2015).

Para reverter este cenário, uma estratégia bastante utilizada é introduzir políticas de Gestão de Demanda de Viagens (GDV, do inglês Travel Demand Management, TDM) na cultura das corporações, conhecido como mobilidade corporativa. Este conceito visa a racionalizar o uso do automóvel nos deslocamentos ao trabalho e, assim, beneficiar funcionários, a cidade e as próprias organizações (Petzhold e Lindau, 2015).

Embora as organizações não controlem a forma como seus funcionários vão ao trabalho, elas podem estimular a mudança de hábitos de deslocamento ao prover informações e incentivos para o uso de modos de transporte mais 


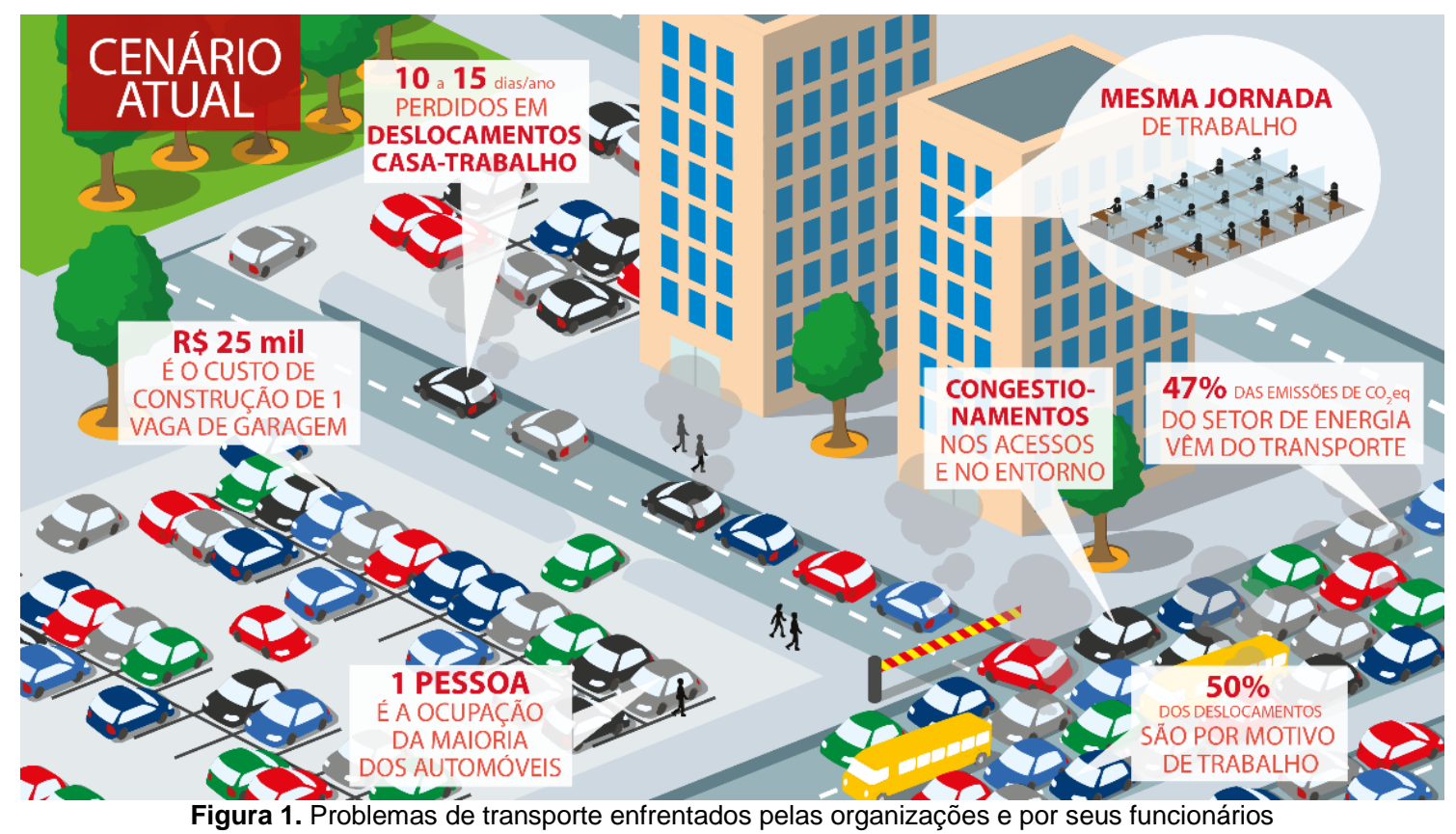

sustentáveis e eficientes em relação ao automóvel. Por exemplo, ao prover auxílio combustível ou vaga de estacionamento grátis para os funcionários e descontar os $6 \%$ do contracheque referente ao Vale Transporte, a organização incentiva a utilização do automóvel em detrimento do transporte coletivo. Através da adoção de um plano de mobilidade corporativa, as corporações são induzidas a revisar as atuais políticas internas existentes e a apoiar hábitos mais sustentáveis e eficientes de deslocamentos ao trabalho, equilibrando os incentivos dados à utilização de todos os modos (Cairns et al., 2010; New Zealand, 2011).

Um plano de mobilidade corporativa é composto por ações que estimulam, por exemplo, a caminhada, a bicicleta, o transporte coletivo e a otimização do uso do automóvel (carona e carsharing) (United Kingdom, 2008). Políticas de mudança no horário da jornada de trabalho (horário flexível, horário escalonado) também podem estar inseridas no plano, bem como medidas capazes de eliminar a necessidade de realizar o deslocamento em si (teletrabalho, vídeo conferência). Dependendo das estratégias adotadas, planos de mobilidade corporativa têm o potencial de reduzir entre 10 e $24 \%$ o número de viagens de automóvel com um único ocupante (Ireland, 2011; United Kingdom, 2008).

O tema ainda é incipiente no Brasil, apesar de já estar consolidado em muitos países. Na literatura acadêmica nacional, existem poucos relatos sobre o assunto (DeCastro, 2014; Petzhold, 2016; Petzhold e Lindau, 2015; Salas et al., 2015). Este artigo visa a trazer e difundir o conceito em âmbito nacional, além de analisar e propor um método para a construção de planos de mobilidade corporativa que se adeque à realidade local.

\section{MÉTODOS ANALISADOS}

A elaboração de planos de mobilidade corporativa é obrigatória por parte de alguns países e cidades ao redor do mundo, por exemplo, em cidades dos Estados Unidos, Canadá, Reino Unido, França, Austrália, Nova Zelândia, entre outros. Esses planos têm o potencial de reduzir o número de deslocamentos em automóvel com um único ocupante, incentivar o uso de meios de transporte mais sustentáveis e, consequentemente, reduzir a emissão de poluentes e gases do efeito estufa. Em razão destes benefícios, pode-se encontrar na literatura internacional alguns métodos já consolidados.

Foram identificados 16 métodos que auxiliam na elaboração do plano. Destes, 13 guiam sua construção através de um roteiro pré-determinado e foram analisados mais detalhadamente. Estes métodos variam quanto a sua estrutura em (i) número de etapas e (ii) atividades previstas (Tabela 1). Entre os principais pontos que podem ser observados estão:

- O processo de desenvolvimento do plano está dividido entre 3 a 8 etapas;

- A maioria dos métodos possui uma etapa inicial de preparação (conquistar apoio da diretoria, designar equipe responsável) antes de iniciar a coleta de dados;

- Todos apontam que é essencial ter o apoio da diretoria. Para 12 dos 13 avaliados, isso deve ser feito já na primeira etapa;

- A maioria dos métodos designa um coordenador para o plano de mobilidade corporativa. Muitos ainda indicam um comitê gestor para auxiliar no processo;

- Todos os métodos coletam dados sobre o padrão de deslocamento de viagens e realizam auditorias no local de trabalho (levantamento de informações relativas à oferta de transporte);

- Apenas metade indica explicitamente a necessidade de alocação de verbas para o desenvolvimento e a implementação das ações do plano;

- Não há consenso quanto à definição de objetivos e metas para o plano, podendo tanto ser realizada antes quanto depois da coleta de dados;

- Ações de marketing e comunicação não estão presentes em cinco dos métodos analisados. Sua inserção dentro do processo de desenvolvimento, normalmente, está após a coleta de dados (diagnóstico) ou junto à implementação das estratégias do plano. 
Tabela 1. Estruturação dos métodos analisados

\begin{tabular}{|c|c|c|c|c|c|c|c|}
\hline Método & Bracknell (2011) & Canada (2010) & Healthy Air (2009) & iCommute (2010) & Ireland (2011) & New Zealand (2011) & NSW (2010) \\
\hline Passo 1 & Obtendo a base certa & Iniciar & $\begin{array}{l}\text { Pesquisa de viagem dos } \\
\text { funcionários }\end{array}$ & $\begin{array}{l}\text { Objetivos, necessidades } \\
\text { e suporte }\end{array}$ & Pré-requisitos & Fase de configuração & $\begin{array}{l}\text { Concordar com } \\
\text { a visão }\end{array}$ \\
\hline Atividades & $\begin{array}{l}\text { Conquistar o apoio da } \\
\text { diretoria e dos funcio- } \\
\text { nários; definir comitê } \\
\text { gestor e coordenador } \\
\text { do plano; realizar diag- } \\
\text { nóstico da organização }\end{array}$ & $\begin{array}{l}\text { Identificar a necessi- } \\
\text { dade; conquistar o } \\
\text { apoio da diretoria; } \\
\text { envolver parcerias; } \\
\text { definir comitê gestor } \\
\text { e coordenador do } \\
\text { plano, alocar verba } \\
\end{array}$ & $\begin{array}{l}\text { Identificar o padrão de } \\
\text { deslocamento dos } \\
\text { funcionários }\end{array}$ & $\begin{array}{l}\text { Identificar a necessidade; } \\
\text { conquistar o apoio da } \\
\text { diretoria; definir objeti- } \\
\text { vos e metas, realizar diag- } \\
\text { nóstico da organização }\end{array}$ & $\begin{array}{l}\text { Conquistar o apoio da } \\
\text { diretoria; definir co- } \\
\text { mitê gestor e coorde- } \\
\text { nador do plano }\end{array}$ & $\begin{array}{l}\text { Conquistar o apoio da dire- } \\
\text { toria e dos funcionários; de- } \\
\text { finir comitê gestor e coor- } \\
\text { denador do plano; alocar } \\
\text { verba; definir escopo do } \\
\text { plano; desenvolver estraté- } \\
\text { gia de comunicação }\end{array}$ & $\begin{array}{l}\text { Conquistar o apoio da } \\
\text { diretoria; definir coor- } \\
\text { denador do plano; defi- } \\
\text { nir uma visão }\end{array}$ \\
\hline Passo 2 & $\begin{array}{l}\text { Concordando com a } \\
\text { visão }\end{array}$ & Avaliar a situação & Auditoria local & Elaborando o plano & $\begin{array}{l}\text { Revisar os padrões } \\
\text { de viagem e políticas }\end{array}$ & Coleta de dados & Definir objetivos \\
\hline Atividades & $\begin{array}{l}\text { Analisar dados; definir } \\
\text { escopo do plano; } \\
\text { definir estratégias }\end{array}$ & $\begin{array}{l}\text { Realizar diagnóstico } \\
\text { da organização; } \\
\text { analisar dados }\end{array}$ & $\begin{array}{l}\text { Avaliar políticas da orga- } \\
\text { nização e o local de } \\
\text { trabalho }\end{array}$ & $\begin{array}{l}\text { Definir estratégias; alocar } \\
\text { verba; definir programa } \\
\text { de ação }\end{array}$ & $\begin{array}{l}\text { Realizar o diagnóstico } \\
\text { da organização; } \\
\text { analisar dados }\end{array}$ & $\begin{array}{l}\text { Realizar o diagnóstico da } \\
\text { organização; analisar dados; } \\
\text { divulgar o diagnóstico }\end{array}$ & $\begin{array}{l}\text { Definir objetivos e } \\
\text { metas }\end{array}$ \\
\hline Passo 3 & $\begin{array}{l}\text { Implementação e } \\
\text { promoção }\end{array}$ & $\begin{array}{l}\text { Criar um plano de } \\
\text { ação }\end{array}$ & Planejamento & $\begin{array}{l}\text { Implementação } \\
\text { e avaliação }\end{array}$ & $\begin{array}{c}\text { Identificar e } \\
\text { implementar ações }\end{array}$ & Programa de ação & $\begin{array}{c}\text { Quantificar o } \\
\text { problema }\end{array}$ \\
\hline Atividades & $\begin{array}{l}\text { Definir programa de } \\
\text { ação; lançar o plano; } \\
\text { implementar a estraté- } \\
\text { gia; implementar ações } \\
\text { de comunicação }\end{array}$ & $\begin{array}{l}\text { Definir objetivos, es- } \\
\text { tratégias, e programa } \\
\text { de ação }\end{array}$ & $\begin{array}{l}\text { Analisar dados; definir } \\
\text { comitê gestor; alocar } \\
\text { verba; definir metas }\end{array}$ & $\begin{array}{l}\text { Lançar, promover e moni- } \\
\text { torar o plano }\end{array}$ & $\begin{array}{l}\text { Analisar dados; defi- } \\
\text { nir programa de ação; } \\
\text { implementar } \\
\text { a estratégia }\end{array}$ & $\begin{array}{l}\text { Definir programa de ação; } \\
\text { definir objetivos e metas; } \\
\text { aprovar o plano }\end{array}$ & $\begin{array}{c}\text { Realizar o diagnóstico } \\
\text { da organização }\end{array}$ \\
\hline Passo 4 & $\begin{array}{l}\text { Monitoramento } \\
\text { e revisão }\end{array}$ & Implementar ações & Liderança & - & Monitorar o plano & Medidas e iniciativas & Desenhar estratégia \\
\hline Atividades & $\begin{array}{l}\text { Monitorar e revisar o } \\
\text { plano }\end{array}$ & $\begin{array}{c}\text { Implementar a estra- } \\
\text { tégia; implementar } \\
\text { ações de comunica- } \\
\text { ção } \\
\end{array}$ & $\begin{array}{l}\text { Conquistar o apoio da di- } \\
\text { retoria; definir coordena- } \\
\text { dor do plano }\end{array}$ & 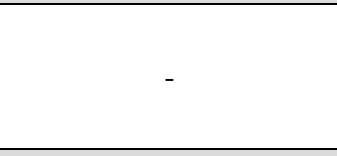 & $\begin{array}{l}\text { Monitorar o plano; } \\
\text { implementar ações } \\
\text { de comunicação }\end{array}$ & Definir estratégias & $\begin{array}{l}\text { Analisar dados; } \\
\text { definir estratégias }\end{array}$ \\
\hline Passo 5 & - & Avaliar resultados & Estratégias & - & - & Marketing e sensibilização & Detalhar iniciativas \\
\hline Atividades & - & Monitorar o plano & Definir estratégias & - & - & $\begin{array}{l}\text { Implementar ações } \\
\text { de comunicação }\end{array}$ & $\begin{array}{l}\text { Definir programa de } \\
\text { ação }\end{array}$ \\
\hline Passo 6 & - & Atualizar o plano & Participação & - & - & Implementação & Monitorar e revisar \\
\hline Atividades & - & Revisar o plano & Monitorar o plano & - & - & $\begin{array}{c}\text { Lançar o plano e implemen- } \\
\text { tar a estratégia }\end{array}$ & $\begin{array}{l}\text { Monitorar e revisar o } \\
\text { plano }\end{array}$ \\
\hline Passo 7 & - & - & - & - & - & $\begin{array}{c}\text { Gerenciando o momento e } \\
\text { monitorando }\end{array}$ & - \\
\hline Atividades & - & - & - & - & - & Monitorar e revisar o plano & - \\
\hline
\end{tabular}


Tabela 1. Estruturação dos métodos analisados (continuação)

\begin{tabular}{|c|c|c|c|c|c|c|}
\hline Método & Perth (2012) & Pollution Probe (2005) & Smart Travel (2010) & Suéscun et al. (2011) & United Kingdom (2008) & Wokingham (2011) \\
\hline Passo 1 & $\begin{array}{c}\text { Construir suporte e definir } \\
\text { escopo do plano }\end{array}$ & Pré-requisitos & Pré-requisitos & Pré-requisitos & Estabelecendo as bases & $\begin{array}{c}\text { Assegurar apoio da } \\
\text { diretoria }\end{array}$ \\
\hline Atividades & $\begin{array}{c}\text { Conquistar o apoio da direto- } \\
\text { ria; anunciar o compromisso; } \\
\text { definir comitê gestor e coor- } \\
\text { denador do plano }\end{array}$ & $\begin{array}{l}\text { Conquistar o apoio da direto- } \\
\text { ria; definir coordenador do } \\
\text { plano }\end{array}$ & $\begin{array}{l}\text { Conquistar o apoio da diretoria; } \\
\text { definir comitê gestor e coorde- } \\
\text { nador do plano; alocar verba }\end{array}$ & $\begin{array}{c}\text { Conquistar o apoio da } \\
\text { diretoria; definir comitê } \\
\text { gestor e coordenador do } \\
\text { plano }\end{array}$ & $\begin{array}{l}\text { Conquistar o apoio da diretoria } \\
\text { e dos funcionários; definir } \\
\text { comitê gestor e coordenador } \\
\text { do plano; alocar verba; definir } \\
\text { escopo do plano; buscar } \\
\text { cooperação } \\
\end{array}$ & $\begin{array}{l}\text { Conquistar o apoio } \\
\text { da diretoria }\end{array}$ \\
\hline Passo 2 & Coletar dados & Análise interna & Pesquisa & Diagnóstico & Coletando informações & $\begin{array}{l}\text { Identificar papéis e } \\
\text { responsabilidades }\end{array}$ \\
\hline Atividades & $\begin{array}{l}\text { Realizar o diagnóstico da or- } \\
\text { ganização; analisar dados; } \\
\text { divulgar diagnóstico }\end{array}$ & $\begin{array}{l}\text { Realizar o diagnóstico da } \\
\text { organização }\end{array}$ & $\begin{array}{l}\text { Realizar o diagnóstico da } \\
\text { organização; analisar dados }\end{array}$ & $\begin{array}{l}\text { Realizar o diagnóstico da } \\
\text { organização }\end{array}$ & $\begin{array}{l}\text { a Realizar o diagnóstico da orga- } \\
\text { nização; analisar dados }\end{array}$ & $\begin{array}{c}\text { Definir comitê } \\
\text { gestor e coordenador } \\
\text { do plano }\end{array}$ \\
\hline Passo 3 & Consultar atores chave & Definição de objetivos & Selecionar ações & $\begin{array}{r}\text { Formulação } \\
\text { de propostas }\end{array}$ & Estabelecendo metas & $\begin{array}{c}\text { Avaliar o local de } \\
\text { trabalho }\end{array}$ \\
\hline Atividades & $\begin{array}{l}\text { Identificar atores chave; } \\
\text { identificar barreiras e } \\
\text { oportunidades }\end{array}$ & $\begin{array}{l}\text { Definir objetivos, metas e in- } \\
\text { dicadores; alocar verba }\end{array}$ & $\begin{array}{l}\text { Definir comitê gestor e coorde- } \\
\text { nador do plano; definir e imple- } \\
\text { mentar a estratégia }\end{array}$ & $\begin{array}{l}\text { Definir estratégias, } \\
\text { metas e indicadores }\end{array}$ & $\begin{array}{l}\text { Definir estratégias, metas; } \\
\text { monitorar o plano }\end{array}$ & $\begin{array}{c}\text { Avaliar políticas da } \\
\text { organização e o local } \\
\text { de trabalho }\end{array}$ \\
\hline Passo 4 & Preparar e revisar o plano & Implementação & Marketing & $\begin{array}{c}\text { Comunicação de } \\
\text { estratégias }\end{array}$ & Marketing & $\begin{array}{l}\text { Realizar pesquisa } \\
\text { com os funcionários }\end{array}$ \\
\hline Atividades & $\begin{array}{l}\text { Definir visão e objetivos; } \\
\text { definir estratégias; definir } \\
\text { programa de ação } \\
\end{array}$ & $\begin{array}{c}\text { Definir estratégias e o pro- } \\
\text { grama de ação; implementar } \\
\text { estratégias }\end{array}$ & $\begin{array}{l}\text { Desenvolver e implementar } \\
\text { ações de comunicação; } \\
\text { monitorar o plano }\end{array}$ & $\begin{array}{c}\text { Desenvolver e } \\
\text { implementar ações } \\
\text { de comunicação } \\
\end{array}$ & $\begin{array}{l}\text { Desenvolver e implementar } \\
\text { ações de comunicação }\end{array}$ & $\begin{array}{c}\text { Identificar o padrão } \\
\text { de deslocamento dos } \\
\text { funcionários } \\
\end{array}$ \\
\hline Passo 5 & $\begin{array}{c}\text { Aprovar e promover o } \\
\text { plano }\end{array}$ & Promoção & - & Implementação & Estratégias & $\begin{array}{c}\text { Identificar } \\
\text { objetivos e metas }\end{array}$ \\
\hline Atividades & Aprovar e lançar o plano & $\begin{array}{c}\text { Desenvolver estratégia de } \\
\text { comunicação; lançar o plano }\end{array}$ & - & Implementar estratégias & Definir estratégias & $\begin{array}{c}\text { Definir objetivos e } \\
\text { metas }\end{array}$ \\
\hline Passo 6 & $\begin{array}{c}\text { Implementar, monitorar e } \\
\text { sustentar }\end{array}$ & Mensuração e avaliação & - & Avaliação & - & $\begin{array}{c}\text { Identificar medidas } \\
\text { e iniciativas }\end{array}$ \\
\hline Atividades & $\begin{array}{l}\text { Implementar a estratégia; } \\
\text { monitorar e revisar o plano }\end{array}$ & Monitorar e revisar o plano & - & Monitorar o plano & - & Definir estratégias \\
\hline Passo 7 & - & - & - & - & - & $\begin{array}{c}\text { Promover e } \\
\text { divulgar }\end{array}$ \\
\hline Atividades & - & - & - & - & - & $\begin{array}{c}\text { Desenvolver e } \\
\text { implementar ações } \\
\text { de comunicação }\end{array}$ \\
\hline Passo 8 & - & - & - & - & - & Monitorar e revisar \\
\hline Atividades & - & - & - & - & - & $\begin{array}{c}\text { Monitorar e revisar o } \\
\text { plano }\end{array}$ \\
\hline
\end{tabular}




\section{NOVO MÉTODO PROPOSTO}

A partir da comparação das estruturas e dos principais pontos destacados pelos métodos analisados, fundamentou-se um novo roteiro para o desenvolvimento de planos de mobilidade corporativa (Tabela 2). Embora os métodos de $\mathrm{Ca}-$ nada (2010) e New Zealand (2011) apresentem bastante semelhança com a nova proposta, foi necessário adequar a proposta para a realidade local, onde a discussão sobre o tema ainda é incipiente e não existem políticas públicas que fomentem a elaboração e implementação deste tipo de plano, implicando a inclusão de novas atividades.

\subsection{O passo a passo}

O método proposto apresenta, sob a forma de um passo a passo, um conjunto de ações que precisam ser desenvolvidas para a construção bem sucedida de um plano de mobilidade corporativa. $\mathrm{O}$ roteiro compreende sete passos principais e suas respectivas atividades (totalizando 26), que possuem tarefas específicas a serem executadas (Figura 2). Os passos, assim como seus principais objetivos, são descritos a seguir:

- Preparação: identificar a necessidade pela qual a organização deseja desenvolver o plano de mobilidade corporativa. Neste passo, deve-se firmar o compromisso da diretoria, mapear quais são os setores chave que devem estar envolvidos com o projeto e definir um comitê gestor e um coordenador responsável por liderar as atividades subsequentes;

- Definição do escopo: definir a visão, os objetivos e as metas que se espera alcançar com o desenvolvimento do plano;

- Comunicação: para conseguir a mudança de comportamento almejada é necessário traçar uma estratégia de comunicação para todo o processo de construção do plano. É nesta etapa também que se deve anunciar para todos os funcionários o compromisso da instituição em desenvolver esta nova política;
- Diagnóstico: entender as circunstâncias que influenciam os deslocamentos ao local de trabalho para a elaboração do plano - inferir os hábitos de deslocamento dos funcionários, quais incentivos os fariam mudar sua escolha modal, avaliar a oferta de transporte do entorno, as políticas da organização que podem influenciar o plano. Estas informações devem ser analisadas, e os resultados comunicados aos funcionários. Para a realização destas atividades, pode ser necessário buscar por instituições que deem suporte técnico;

- Elaboração: reavaliar as metas definidas tendo em vista os resultados do diagnóstico e definir os indicadores de desempenho que traduzem o sucesso do plano. É nesta etapa que se alocam os recursos para a implementação do plano. As medidas a serem incentivadas devem ser enquadradas ao orçamento disponível. É importante definir um programa de ação que contenha um cronograma prevendo a implementação de cada uma delas;

- Implementação e promoção: assegurar que o plano opere em seu pleno potencial. É importante prever incentivos e promoções para manter os funcionários engajados e constantemente informados de cada uma das medidas de mobilidade corporativa implementadas. Pode ser necessário buscar parcerias para suporte técnico;

- Monitoramento e revisão: para garantir que as medidas e iniciativas alcancem as metas e objetivos propostos, é necessário monitorar regularmente o desempenho do plano. Dependendo dos resultados, pode ser necessário revisar e atualizar o plano.

O conteúdo e o processo de desenvolvimento de um plano de mobilidade corporativa propostos são flexíveis. Algumas atividades podem ocorrer de forma simultânea ou em uma ordem diferente, contudo é recomendável que se realizem todos os passos e atividades. Cada organização pode adaptar o método conforme as circunstâncias locais, no entanto, sempre devem refletir os princípios identificados neste roteiro.

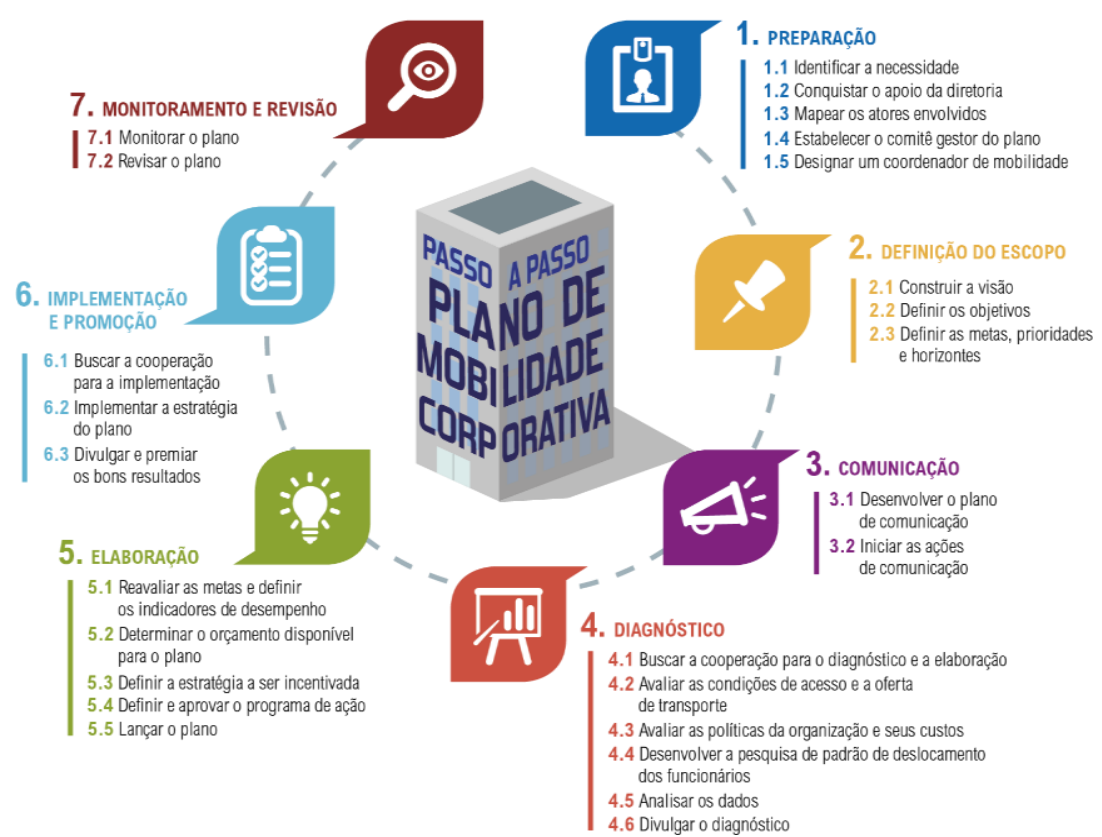

Figura 2. Método proposto para o desenvolvimento de planos de mobilidade corporativa 
Tabela 2. Coincidência entre o método proposto e os existentes

\begin{tabular}{|c|c|c|c|c|c|c|c|c|c|c|c|c|c|c|}
\hline Passo a passo proposto & Bracknell & Canada & $\begin{array}{l}\text { Healthy } \\
\text { Air }\end{array}$ & iCommute & Ireland & $\begin{array}{c}\text { New } \\
\text { Zealand }\end{array}$ & NSW & Perth & $\begin{array}{c}\text { Pollution } \\
\text { Probe }\end{array}$ & $\begin{array}{l}\text { Smart } \\
\text { Travel }\end{array}$ & $\begin{array}{l}\text { Suéscun } \\
\text { et al. }\end{array}$ & $\begin{array}{l}\text { United } \\
\text { Kingdom }\end{array}$ & Wokingham & $\begin{array}{c}\% \text { de } \\
\text { métodos } \\
\text { com a } \\
\text { atividade }\end{array}$ \\
\hline \multicolumn{15}{|l|}{ 1) Preparação } \\
\hline Identificar a necessidade & & $\mathrm{x}$ & & $\mathrm{x}$ & & & & & & & & & & $15,4 \%$ \\
\hline Conquistar o apoio da diretoria & $\mathrm{x}$ & $\mathrm{x}$ & $\mathrm{x}$ & $\mathrm{x}$ & $\mathrm{x}$ & $\mathrm{x}$ & $\mathrm{x}$ & $\mathrm{x}$ & $\mathrm{x}$ & $\mathrm{x}$ & $\mathrm{x}$ & $\mathrm{x}$ & $\mathrm{x}$ & $100 \%$ \\
\hline Mapear os atores envolvidos & & & & & & & & & & & & & & $0 \%$ \\
\hline $\begin{array}{l}\text { Estabelecer o comitê gestor do } \\
\text { plano }\end{array}$ & $\mathrm{x}$ & $\mathrm{x}$ & $\mathrm{x}$ & & $\mathrm{x}$ & $\mathrm{x}$ & & $\mathrm{x}$ & & $\mathrm{x}$ & $\mathrm{x}$ & $\mathrm{x}$ & $\mathrm{x}$ & $76,9 \%$ \\
\hline $\begin{array}{l}\text { Designar um coordenador de } \\
\text { mobilidade }\end{array}$ & $\mathrm{x}$ & $\mathrm{x}$ & $\mathrm{x}$ & & $\mathrm{x}$ & $\mathrm{x}$ & $\mathrm{x}$ & $\mathrm{x}$ & $\mathrm{x}$ & $\mathrm{x}$ & $\mathrm{x}$ & $\mathrm{x}$ & $\mathrm{x}$ & $92,3 \%$ \\
\hline \multicolumn{15}{|l|}{ 2) Definição de escopo } \\
\hline Construir a visão & $\mathrm{x}$ & & & & & & $\mathrm{x}$ & & & & & & & $15,4 \%$ \\
\hline Definir os objetivos & $\mathrm{x}$ & $\mathrm{x}$ & & $\mathrm{x}$ & & $\mathrm{x}$ & $\mathrm{x}$ & $\mathrm{x}$ & $\mathrm{x}$ & & & $\mathrm{x}$ & $\mathrm{x}$ & $69,2 \%$ \\
\hline $\begin{array}{l}\text { Definir as metas, prioridades e } \\
\text { horizontes }\end{array}$ & $\mathrm{x}$ & $\mathrm{x}$ & $\mathrm{x}$ & $\mathrm{x}$ & $\mathrm{x}$ & $\mathrm{x}$ & $\mathrm{x}$ & $\mathrm{x}$ & $\mathrm{x}$ & & $\mathrm{x}$ & $\mathrm{x}$ & $\mathrm{x}$ & $\mathbf{9 2 , 3 \%}$ \\
\hline \multicolumn{15}{|l|}{ 3) Comunicação } \\
\hline $\begin{array}{l}\text { Desenvolver o plano de } \\
\text { comunicação }\end{array}$ & $\mathrm{x}$ & $\mathrm{x}$ & & & & $\mathrm{x}$ & & & $\mathrm{x}$ & $\mathrm{x}$ & $\mathrm{x}$ & $\mathrm{x}$ & $\mathrm{x}$ & $61,5 \%$ \\
\hline Iniciar as ações de comunicação & & & & & & $\mathrm{x}$ & & $\mathrm{x}$ & & & & & & $15,4 \%$ \\
\hline \multicolumn{15}{|l|}{ 4) Diagnóstico } \\
\hline $\begin{array}{l}\text { Buscar a cooperação para } \\
\text { diagnóstico e elaboração }\end{array}$ & & $\mathrm{x}$ & & & & & & & & & & $\mathrm{x}$ & & $15,4 \%$ \\
\hline $\begin{array}{l}\text { Avaliar as condições de acesso e } \\
\text { a oferta de transporte }\end{array}$ & $\mathrm{x}$ & $\mathrm{x}$ & $\mathrm{x}$ & $\mathrm{x}$ & $\mathrm{x}$ & $\mathrm{x}$ & & $\mathrm{x}$ & & & $\mathrm{x}$ & $\mathrm{x}$ & $\mathrm{x}$ & $76,9 \%$ \\
\hline $\begin{array}{l}\text { Avaliar as políticas da organiza- } \\
\text { ção e seus custos }\end{array}$ & $\mathrm{x}$ & $\mathrm{x}$ & $\mathrm{x}$ & $\mathrm{x}$ & $\mathrm{x}$ & $\mathrm{x}$ & $\mathrm{x}$ & $\mathrm{x}$ & $\mathrm{x}$ & $\mathrm{x}$ & $\mathrm{x}$ & $\mathrm{x}$ & $\mathrm{x}$ & $100 \%$ \\
\hline $\begin{array}{l}\text { Desenvolver a pesquisa de } \\
\text { padrão de deslocamento dos } \\
\text { funcionários }\end{array}$ & $\mathrm{x}$ & $\mathrm{x}$ & $\mathrm{x}$ & $\mathrm{x}$ & $\mathrm{x}$ & $\mathrm{x}$ & $\mathrm{x}$ & $\mathrm{x}$ & $\mathrm{x}$ & $\mathrm{x}$ & $\mathrm{x}$ & $\mathrm{x}$ & $\mathrm{x}$ & $100 \%$ \\
\hline Analisar os dados & $\mathrm{x}$ & $\mathrm{x}$ & $\mathrm{x}$ & & $\mathrm{x}$ & $\mathrm{x}$ & $\mathrm{x}$ & $\mathrm{x}$ & & $\mathrm{x}$ & & $\mathrm{x}$ & & $69,2 \%$ \\
\hline Divulgar o diagnóstico & & & & & & & & $\mathrm{x}$ & & & & & & $7,7 \%$ \\
\hline
\end{tabular}


Tabela 2. Coincidência entre o método proposto e os existentes (continuação)

\begin{tabular}{|c|c|c|c|c|c|c|c|c|c|c|c|c|c|c|}
\hline Passo a passo proposto & Bracknell & Canada & $\begin{array}{l}\text { Healthy } \\
\text { Air }\end{array}$ & iCommute & Ireland & $\begin{array}{l}\text { New } \\
\text { Zealand }\end{array}$ & NSW & Perth & $\begin{array}{l}\text { Pollution } \\
\text { Probe }\end{array}$ & $\begin{array}{l}\text { Smart } \\
\text { Travel }\end{array}$ & $\begin{array}{l}\text { Suéscun } \\
\text { et al. }\end{array}$ & $\begin{array}{l}\text { United } \\
\text { Kingdom }\end{array}$ & Wokingham & $\begin{array}{l}\% \text { de } \\
\text { métodos } \\
\text { com a } \\
\text { atividade }\end{array}$ \\
\hline \multicolumn{15}{|l|}{ 5) Elaboração } \\
\hline $\begin{array}{l}\text { Reavaliar as metas e definir os } \\
\text { indicadores de desempenho }\end{array}$ & $\mathrm{x}$ & $\mathrm{x}$ & $\mathrm{x}$ & $\mathrm{x}$ & $\mathrm{x}$ & $\mathrm{x}$ & & $\mathrm{x}$ & $\mathrm{x}$ & & $\mathrm{x}$ & $\mathrm{x}$ & $\mathrm{x}$ & $84,6 \%$ \\
\hline $\begin{array}{l}\text { Determinar o orçamento disponível } \\
\text { para o plano }\end{array}$ & & $\mathrm{x}$ & $\mathrm{x}$ & $\mathrm{x}$ & & $\mathbf{x}$ & & & $\mathrm{x}$ & $\mathrm{x}$ & & $\mathrm{x}$ & & $53,8 \%$ \\
\hline Definir a estratégia a ser incentivada & $\mathrm{x}$ & $\mathrm{x}$ & $\mathrm{x}$ & $\mathrm{x}$ & $\mathrm{x}$ & $\mathrm{x}$ & $\mathrm{x}$ & $\mathrm{x}$ & $\mathrm{x}$ & $\mathrm{x}$ & $\mathrm{x}$ & $\mathrm{x}$ & $\mathrm{x}$ & $100 \%$ \\
\hline Definir e aprovar o programa de ação & $\mathrm{x}$ & $\mathrm{x}$ & & $\mathrm{x}$ & $\mathrm{x}$ & $\mathrm{x}$ & $\mathrm{x}$ & $\mathrm{x}$ & $\mathrm{x}$ & & & & & $61,5 \%$ \\
\hline Lançar o plano & $\mathrm{x}$ & & & $\mathrm{x}$ & & $\mathrm{x}$ & & $\mathrm{x}$ & $\mathrm{x}$ & & & & & $38,5 \%$ \\
\hline \multicolumn{15}{|l|}{ 6) Implementação e promoção } \\
\hline $\begin{array}{l}\text { Buscar a cooperação para } \\
\text { implementação }\end{array}$ & & $\mathrm{x}$ & & & & & & & & & & $\mathrm{x}$ & & $15,4 \%$ \\
\hline Implementar a estratégia do plano & $\mathrm{x}$ & $\mathrm{x}$ & & & $\mathrm{x}$ & $\mathrm{x}$ & & $\mathrm{x}$ & $\mathrm{x}$ & $\mathrm{x}$ & $\mathrm{x}$ & & $\mathrm{x}$ & $69,2 \%$ \\
\hline Divulgar e premiar os bons resultados & & $\mathrm{x}$ & & $\mathrm{x}$ & $\mathrm{x}$ & $\mathrm{x}$ & & & $\mathrm{x}$ & $\mathrm{x}$ & & $\mathrm{x}$ & $\mathrm{x}$ & $61,5 \%$ \\
\hline \multicolumn{15}{|l|}{ 7) Monitoramento e revisão } \\
\hline Monitorar o plano & $\mathrm{x}$ & $\mathrm{x}$ & $\mathrm{x}$ & $\mathrm{x}$ & $\mathrm{x}$ & $\mathrm{x}$ & $\mathrm{x}$ & $\mathrm{x}$ & $\mathrm{x}$ & $\mathrm{x}$ & $\mathrm{x}$ & $\mathrm{x}$ & $\mathrm{x}$ & $100 \%$ \\
\hline Revisar o plano & $\mathrm{x}$ & $\mathrm{x}$ & & & & $\mathrm{x}$ & $\mathrm{x}$ & $\mathrm{x}$ & $\mathrm{x}$ & & & & $\mathrm{x}$ & $53,8 \%$ \\
\hline \% de atividades coincidentes & $69,2 \%$ & $80,8 \%$ & $46,2 \%$ & $53,8 \%$ & $53,8 \%$ & $76,9 \%$ & $46,2 \%$ & $69,2 \%$ & $61,5 \%$ & $46,2 \%$ & $46,2 \%$ & $65,4 \%$ & $57,7 \%$ & - \\
\hline
\end{tabular}




\subsection{Principais diferenciais}

A partir da comparação dos métodos analisados, identificaram-se seis principais pontos que necessitaram ser modificados ou incorporados ao processo de construção do plano de mobilidade corporativa, a fim de enquadrá-lo dentro da realidade local. As adequações feitas são descritas a seguir.

\subsubsection{Identificação da necessidade}

Muitos dos métodos analisados contêm menções aos benefícios oriundos da implantação de um plano de mobilidade corporativa. Contudo, apenas dois (Canada, 2010; iCommute, 2010) incluem a identificação da necessidade como parte constituinte do processo de desenvolvimento do plano. Identificar quaisquer obrigações, desafios e oportunidades relacionados a transporte é essencial para justificar a elaboração do plano de mobilidade corporativa. Por exemplo, deve-se buscar informações sobre gargalos relacionados a transportes, planos de expansão, déficit de vagas de estacionamento e planos para ampliá-las; desejos de melhorar o desempenho em relatórios de sustentabilidade ou melhorar a imagem frente a parceiros.

Questões relacionadas ao bem-estar dos funcionários e a metas de sustentabilidade da organização também devem ser consideradas.

Compreender de forma clara quais são as necessidades da organização e os potenciais ganhos é essencial para estruturar uma boa argumentação sobre as razões pelas quais a instituição deve desenvolver o plano e investir esforços e recursos financeiros e humanos (Canada, 2010, Roby, 2010). Tais argumentos também são necessários para ganhar o suporte de departamentos estratégicos que devem estar envolvidos no projeto

\subsubsection{Mapeamento dos atores envolvidos}

Identificar setores, pessoas ou associações relevantes na construção ou impactadas direta ou indiretamente é fundamental para todo e qualquer projeto. Apesar de 76,9\% dos métodos analisados sugerir a formação de um comitê gestor para o plano de mobilidade corporativa e indicar setoreschave para compô-lo, nenhum auxilia em como identificar estes atores. Por isso, julgou-se importante incorporar uma atividade que pudesse suprir esta carência.

O mapeamento dos atores consiste em classificar os grupos envolvidos em diferentes categorias, de acordo com o seu interesse e sua influência no processo de desenvolvimento do plano. Isso permite planejar melhor o envolvimento e definir como realizar a comunicação com cada um deles (Brasil, 2015; EMBARQ Brasil, 2014; Rao et al., 2013; Wefering et al. 2014). O resultado final deste processo é uma matriz que reflete a maior ou menor relevância dos atores. Os atores com alta influência e alto interesse devem estar plenamente envolvidos na elaboração do plano e por isso devem ter representantes dentro do comitê gestor que conduzirá seu processo de desenvolvimento.

Tipicamente, os atores identificados como mais relevantes dentro da organização pertencem aos setores: de comunicação, financeiro, gestão de frotas, instalações/facilities, recursos humanos e sustentabilidade. Esses atores devem compor um comitê gestor que liderará a execução das próximas etapas do desenvolvimento do plano de mobilidade corporativa. Além disso, representantes de grupos formais ou informais de bicicleta ou carona, por exemplo, bem como a associação dos funcionários devem ser envolvidos no processo. Externo à organização, o poder público se apresenta como peça relevante, pois é necessária a articulação com ele para implementação de medidas que qualifiquem a região onde a organização se situa, por exemplo, calçadas, pontos de parada, entre outros.

\subsubsection{Definição do escopo e reavaliação das metas iniciais}

A comparação entre os métodos existentes evidenciou uma falta de consenso entre a definição do escopo (visão, objetivos e metas) antes ou após a fase de diagnóstico. Sucintamente, a visão pode ser definida como o elemento central que deve expressar o futuro desejado pela organização e pelos seus funcionários para as questões de mobilidade. Já os objetivos devem traduzir o que se pretende obter com o plano, refletindo as necessidades identificadas, as motivações por trás do plano e o que se pretende alcançar. As metas, por sua vez, expressam os objetivos de forma mensurável e permitem a avaliação do progresso em direção ao sucesso. Elas devem atender ao princípio SMART, sendo específicas, mensuráveis, alcançáveis, relevantes e vinculadas ao tempo (TRB, 2005). Um exemplo de escopo é dado a seguir:

- Visão: criar uma cultura e ambiente corporativo onde escolhas mais sustentáveis de deslocamento são ativamente encorajadas

- Objetivo: reduzir a participação do automóvel com um único ocupante na divisão modal da organização - Meta: aumentar em 10\% o número de caronas dentro de dois anos.

Dois dos documentos analisados (iCommute, 2010; NSW, 2010) estabelecem objetivos e metas antes de realizar o diagnóstico da mobilidade do empreendimento, enquanto sete (Bracknell, 2011; Canada, 2010; Healthy Air, 2009; Ireland, 2011; Suéscun et al., 2011; United Kingdom, 2008; Wokingham, 2011) os definem após o diagnóstico. Apenas dois (New Zealand 2011; Perth, 2012) apontam objetivos e metas antes de coletar os dados e os reavaliam tendo em vista os resultados obtidos através da pesquisa de padrão de deslocamento dos funcionários e das demais informações levantadas.

Optou-se por adotar o proposto por New Zealand (2011) e Perth (2012), uma vez que é essencial ter clara a visão, os objetivos e metas do porquê desenvolver um plano de mobilidade corporativa logo no início de seu desenvolvimento. Contudo, também é importante reavaliar as metas anteriormente estabelecidas. Os resultados obtidos provêm uma riqueza de detalhes que pode nortear as metas de forma mais concreta em direção aos desejos dos funcionários e às políticas da organização.

\subsubsection{Protagonismo da comunicação}

Fortes campanhas de comunicação implicam maior utilização de alternativas mais sustentáveis de transporte (United Kingdom, 2008) e, consequentemente, um maior sucesso do plano. Por isso, é natural que $61,5 \%$ dos métodos contemplem o desenvolvimento de uma estratégia de comunicação. 
Contudo, a maioria deles apenas considera que estas campanhas devem ser iniciadas após a elaboração do plano ou, até mesmo, depois da implementação das primeiras estratégias de mobilidade corporativa.

New Zealand (2011) é o único a considerar o desenvolvimento de uma estratégia de comunicação já na fase inicial da construção do plano. United Kingdom (2008) afirma que a comunicação inicia apenas quando se envia a pesquisa de padrão de deslocamento aos funcionários. Por outro lado, Perth (2012) sugere que seja anunciado o compromisso da diretoria da organização com a política de mobilidade corporativa já no primeiro momento como forma de engajar todos os colaboradores. Contudo, os três sugerem que as ações de comunicação só sejam fortemente implementadas após a elaboração do plano.

Um plano de mobilidade corporativa trata de mudar os hábitos de deslocamento das pessoas e seu sucesso depende integralmente da adesão dos funcionários às novas ideias (Canada, 2010; New Zealand, 2011; Perth, 2012; TfL 2008). Por isso o novo método proposto recomenda um maior protagonismo da comunicação e seu desenvolvimento em um estágio mais inicial do processo. Embora seja mais crucial durante a implementação, a comunicação deve ser utilizada sucessivamente para divulgar as novidades e progressos do plano. Ela está presente nas seguintes atividades previstas no método:

- Iniciar ações de comunicação: comunicar o compromisso da diretoria com o desenvolvimento do plano;

- Desenvolver pesquisa de padrão de deslocamento dos funcionários: promover a pesquisa antes, durante e depois do seu período de aplicação para obter uma maior taxa de resposta;

- Divulgar diagnóstico: informar aos funcionários os resultados iniciais inferidos;

- Lançar o plano: divulgar o plano e as ações propostas para a melhoria dos problemas enfrentados em relação ao transporte;

- Implementar as estratégias do plano: divulgar as estratégias de mobilidade corporativa implementadas para fomentar a adesão dos funcionários;

- Divulgar e premiar bons resultados: divulgar histórias e depoimentos de funcionários que mudaram seus hábitos de deslocamento em virtude das estratégias implementadas;

- Monitorar o plano: divulgar a evolução dos indicadores que reflitam a mudança de hábito da organização frente ao cenário inicial.

\subsubsection{Necessidade de cooperação técnica}

A maioria dos métodos avaliados é proveniente de países ou cidades nas quais a elaboração de um plano de mobilidade corporativa é obrigatória para empreendimentos que se enquadrem dentro de um determinado critério, seja número de funcionários ou área construída. Nestes locais, existem as chamadas TMA (Transportation Management Associations), organizações formadas por grupos de organizações que auxiliam em todo o processo de desenvolvimento de planos de mobilidade corporativa e que, normalmente, possuem apoio do governo local (Litman, 2014).

Por essas organizações já existirem desde a década de 1980 (ACT, 2001), é possível que a ‘busca por cooperação’ seja algo implícito nos métodos avaliados. Pelo tema de mobilidade corporativa ser um conceito novo no Brasil, não existe muita expertise sobre o assunto, tanto em termos de corpo técnico quanto de organizações que adotam soluções do gênero e possam partilhar experiências. Dessa forma, optou-se por explicitar a necessidade de buscar cooperação para desenvolver o plano não só durante os passos mais teóricos (diagnóstico e elaboração), mas também durante a implementação das estratégias.

Os parceiros podem aportar conhecimento através de lições aprendidas e estudos de caso. Eles também podem fornecer materiais consolidados que, além de acelerar o processo, aumentam a confiabilidade e possibilitam a comparação (benchmarking) entre organizações que implantaram planos de mobilidade corporativa. $\mathrm{O}$ envolvimento de parceiros externos pode adicionar custos em alguns casos, mas também pode reduzir o esforço pessoal e o tempo despendido (Canada, 2010).

\subsubsection{Coleta de dados sobre custos}

Antes mesmo de existir um plano de mobilidade corporativa, é provável que já exista uma série de políticas corporativas que influenciam os hábitos de deslocamento dos colaboradores. Algumas políticas estimulam viagens mais sustentáveis e eficientes, enquanto outras agem como barreiras ao plano. Elas também são fontes de custos para as organizações.

Apesar de todos os métodos levantarem as políticas adotadas pela organização que possam vir a influenciar as estratégias de mobilidade corporativa a serem adotadas, nenhum menciona o levantamento dos custos relacionados às questões de transporte. Esses fatores devem ser identificados e são oportunidades que devem ser exploradas pelo plano. Embora algumas estratégias possam necessitar de investimentos, outras podem reduzir custos ou, até mesmo, gerar receitas e, assim, subsidiar os incentivos adotados em prol do transporte sustentável (Canada, 2010; New Zealand, 2011; United Kingdom, 2008).

\section{CONSIDERAÇÕES FINAIS}

O setor corporativo possui um papel fundamental na busca por soluções para os problemas relacionados ao transporte, uma vez que grande parte dos deslocamentos diários ocorre por motivo de trabalho. Este artigo visa a introduzir um conceito ainda pouco difundido em âmbito nacional, além de propor um método para a construção de planos de mobilidade corporativa que se adeque à realidade local. Embora as organizações não controlem a forma como seus funcionários vão ao local de trabalho, elas podem estimular a mudança de hábitos de deslocamento ao prover informações e incentivos para o uso de modos de transporte mais sustentáveis e eficientes em relação ao automóvel.

Existem métodos já consolidados no cenário internacional para a construção desses planos, contudo necessitam adequações ao contexto vivenciado no Brasil. Através da comparação de 16 distintos métodos, é proposto um novo método adaptado à realidade local. $\mathrm{O}$ método apresenta, sob a forma de um passo a passo, um conjunto de ações para a construção bem sucedida de um plano de mobilidade corporativa. Este novo método se divide em sete passos: (i) preparação, (ii) definição do escopo, (iii) comunicação, (iv) 
diagnóstico, (v) elaboração, (vi) implementação e promoção e (vii) monitoramento e revisão; totalizando 26 atividades.

O método proposto se diferencia em seis pontos principais frente aos analisados. O primeiro deles é a identificação da necessidade. No Brasil, não há políticas públicas que obriguem organizações a desenvolverem planos de mobilidade corporativa. Por isso é preciso identificar desafios e oportunidades relacionados a transporte que convençam lideranças a investirem nisso. Outro ponto é o mapeamento dos atores envolvidos, que consiste em identificar setores, pessoas ou associações relevantes na construção do plano. O terceiro aspecto é a definição do escopo e reavaliação das metas iniciais, que prevê a revisão das metas tendo em vista os dados obtidos no diagnóstico e, assim, pode nortear a definição de metas mais realistas.

O quarto ponto é o protagonismo da comunicação. Normalmente, seu papel se restringe a ações pontuais, contudo esforços de comunicação são chave para alcançar mudanças de hábito. O quinto aspecto é a necessidade de cooperação técnica. Os métodos analisados provêm de países onde mobilidade corporativa já é uma prática consolidada e diversas instituições e o próprio poder público dão suporte no desenvolvimento dos planos, o que não é o caso brasileiro. Por fim o último ponto é a coleta de dados sobre custos. Políticas corporativas influenciam os hábitos de deslocamento dos funcionários e representam uma fonte de custos para a empresa. Essas informações devem ser coletadas e representam oportunidades que devem ser exploradas no plano, bem como argumentos junto à liderança.

Através deste trabalho, espera-se influenciar organizações a desenvolverem planos de mobilidade corporativa. Regiões com muitos postos de trabalho, distritos/bairros/cidades industriais e universidades representam oportunidades para a aplicação desse tipo de estratégia. Cidades com mais de 20 mil habitantes já são obrigadas a elaborar planos de mobilidade urbana, contudo, regiões que apresentam uma grande concentração de organizações e onde os padrões de deslocamento são mais previsíveis não necessitam fazê-lo. Mudar esta prática pode desencadear uma série de benefícios para a mobilidade urbana das cidades e para a melhoria da qualidade de vida das pessoas.

\section{AGRADECIMENTOS}

Os autores agradecem o apoio do WRI Brasil Cidades Sustentáveis, do WRI Brasil, do Banco Mundial, da Caterpillar Foundation e da Children's Investment Fund Foundation.

\section{REFERÊNCIAS}

ACT (2001) TMA Handbook: a guide to successful transportation management associations. Washington D.C., USA.

Bahia, Secretaria de Infraestrutura, Departamento de Infraestrutura de Transportes da Bahia (2012) Pesquisa de Mobilidade - Região Metropolitana de Salvador: síntese dos resultados. Salvador, Brasil.
Bracknell Forest Council (2011) Travel Choice for Business: a local guide to workplace travel plan. Bracknell Forest, United Kingdom.

Brasil, Ministério das Cidades, Secretaria Nacional de Transporte e da Mobilidade Urbana (2015). PlanMob - Caderno de referência para elaboração de plano de mobilidade urbana. Brasília/DF, Brasil.

Broaddus, A., T. Litman e G. Menon (2009). Transportation Demand Management. GTZ - Deutsche Gesellschaft für Technische Zusamennarbeit, Eschborn, Germany.

Cairns, S., C. Newson, e A. Davis (2010) Understanding Successful Workplace Travel Initiatives in the UK. In:

Transportation Research Part A, Policy and Practice, v. 44, issue 7, p 473-494. doi:10.1016/j.tra.2010.03.010

Canada, Transport Canada (2010) Workplace Travel Plans: guidance for Canadian employers. Ottawa, Canada.

DeCastro, J. (2014) Caracterização e Análise do Deslocamento Casa-Trabalho-Casa em Empresas Localizadas na Barra da Tijuca. Dissertação (Mestrado) - Programa de Pós-graduação em Engenharia de Transportes, Universidade Federal do Rio de Janeiro, Rio de Janeiro.

Distrito Federal, Secretaria de Estado de Transportes (2010) Plano Diretor de Transporte Urbano e Mobilidade do Distrito Federal e Entorno - Relatório final. Brasília/DF, Brasil, outubro 2010.

EMBARQ Brasil (2014) Passo a Passo para a Construção de um Plano de Mobilidade Urbana. Porto Alegre, Brasil.

Disponível em: <http://d.pr/f/1eLcr〉. Acesso em: 08 maio 2015.

Healthy Air (2009) Employer's Guide to a Commute Solutions Program. Dane County, United States.

iCommute (2010) Commuter Benefit Starter Kit for Employers. San Diego, United States.

Ireland, National Transport Authority (2011) Workplace Travel Plans: a guide for implementers. Dublin, Ireland.

Litman, T. (2014) Transportation Management Associations. Disponível em: <http://www.vtpi.org/tdm/ tdm44.htm>. Acesso em: 28 maio 2015 .

Minas Gerais, Secretaria Extraordinária de Gestão Metropolitana, Agência de Desenvolvimento da Região Metropolitana de Belo Horizonte (2013) Pesquisa Origem e Destino 2011-2012 Relatório Completo. Belo Horizonte, Brasil.

NSW (2010) Workplace Travel Guidance. New South Wales, Australia.

New Zealand, NZ Transport Agency (2011) Workplace Travel Plan Guidelines: general travel planning know-how with a focus on travel to and from work. New Zealand.

Observatório do Clima (2015) Evolução das Emissões de Gases de Efeito Estufa no Brasil (1990-2013) - Setor de Energia e Processos Industriais. São Paulo, Brasil.

Pereira, R. H. M. e T. Schwanen (2013) Tempo de Deslocamento Casa-Trabalho no Brasil (1992-2009): diferenças entre regiões metropolitanas, níveis de renda e sexo. Instituto de Pesquisa Econômica Aplicada, Brasilia/DF, Brasil. 
Perth, Department of Environment and Conservation, Department of Transport (2012) TravelSmart to Work: a workplace travel planning guide. Perth, Australia.

Petzhold, G. S. (2016) Mobilidade Corporativa: Como Engajar Organizações Brasileiras em Prol da Melhoria do Transporte Urbano. Dissertação (Mestrado) - Programa de Pós-graduação em Engenharia de Produção, Universidade Federal do Rio Grande do Sul, Porto Alegre.

Petzhold, G. S. e L. A. Lindau (2015) O Papel das Corporações na Busca da Melhoria das Condições de Mobilidade Urbana nas Cidades. In: Anais XXIX Congresso de Pesquisa e Ensino em Transportes, ANPET, Ouro Preto.

Pollution Probe (2005) S-M-A-R-T Movement: trip reduction manual. Canada. Disponível em: <http://www.

pollutionprobe.org/publications/s-m-a-r-t-movement-tripreduction-manual/>. Acesso em: 09 maio 2015.

Porto Alegre, Empresa Pública de Transporte e Circulação (2004) Pesquisa de Origem e Destino de Porto Alegre. Porto Alegre, Brasil.

Rao P., R. King, R. Stanich., T. Pande e C. Dhingra (2013) Quick Guide: how to develop an urban mobility plan. UN Habitat, Nairobi, Kenya.

Rio de Janeiro (Estado), Secretaria de Transportes (2015) Plano Diretor de Transporte Urbano da Região Metropolitana do Rio de Janeiro 2013 - Relatório 4, Planejamento e Execução das Pesquisas, Parte 3: diagnóstico da situação atual. Rio de Janeiro, Brasil.

Roby H. (2010) Workplace travel plans: past, present and future. In: Journal of Transport Geography, v. 18, p 23-30.

doi:10.1016/j.jtrangeo.2008.11.010

Salas, D. C., G. Darido, S. Mehndiratta e A. Leal (2015) A Commuter-Based Traffic Demand Management Approach for Latin America: Results from Voluntary Corporate Mobility Pilots in Sao Paulo and Mexico City. In 94th Transportation Research Board Annual Meeting. Washington D. C., United States, 2015.

São Paulo (Estado), Secretaria dos Transportes Metropolitanos (2013) Pesquisa de Mobilidade da Região Metropolitana de São Paulo 2012: principais resultados pesquisa domiciliar. São Paulo, Brasil.

São Paulo (Estado), Secretaria dos Transportes Metropolitanos (2012) Pesquisa Origem-Destino 2011 - Região Metropolitana de Campinas: síntese dos resultados das pesquisas domiciliar e cordon line. Campinas, Brasil.

Smart Travel Workplaces (2010) Your Step by Step Guide to Travel Plans. Ireland.

Suescún, J. P. B., M. F. O. Carrascal, F. A. Rozo e S. Cucchi (2011) Guía para el Desarrollo de Planes Empresariales de Movilidad Sostenible - PEMS en Bogotá. Universidad de los Andes, Bogotá, Colombia.

TfL (2008) Guidance for Workplace Travel Planning for Development. Transport for London, United Kingdom.

TRB (2005) TCRP 107 - Analyzing the Effectiveness of Commuter Benefits Programs. Transportation Research Board, Washington D. C., United States.

United Kingdon, Department for Transport (2008) The Essential Guide to Travel Planning. London, United Kingdom.
Van Malderen, L., B. Jourquin, I. Thomas, T. Vanoutrive, A. Verhetsel e F. Witlox (2012) On the mobility policies of companies: What are the good practices? The Belgian case. In: Transport Policy, v. 21, p 10-19.

DOI:10.1016/j.tranpol.2011.12.005

Wefering F., S. Rupprecht, S. Bührmann e S. Böhler-Baedeker (2014) Developing and Implementing a Sustainable Urban Mobility Plan. Rupprecht Consult. Brussels, Belgium.

Wokingham Borough Council (2011) WorkPlace Travel Plan Guidance. Wokingham, United Kingdom. 